LA-UR- $05-2818$

Title:

CALIBRATION AND USE OF A RUGGED NEW

PIEZORESISTIVE PRESSURE TRANSDUCER

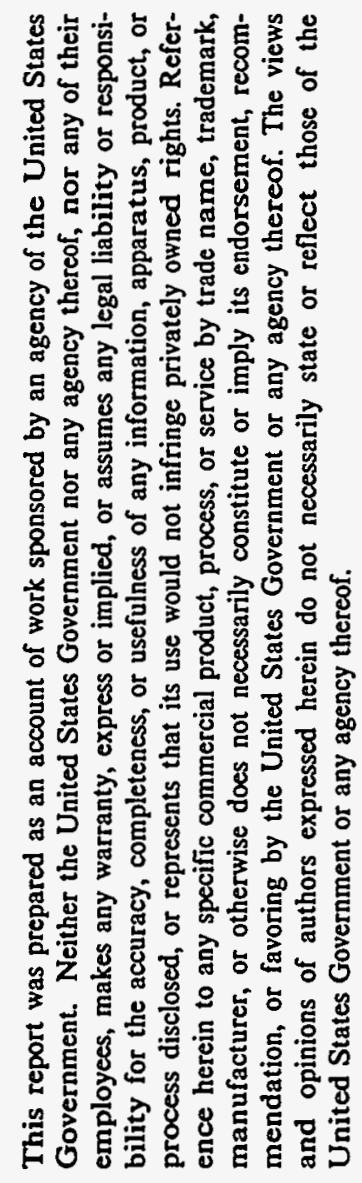
Author(s):
R. A. Lucht
J. A. Charest

Submitted to:
APS Meeting
Seattle, Washington
DISTRIBUTION OF THIS DOCUMENT IS UNLIMIED GH

$$
\begin{aligned}
& 0.92963 \\
& 0.51
\end{aligned}
$$

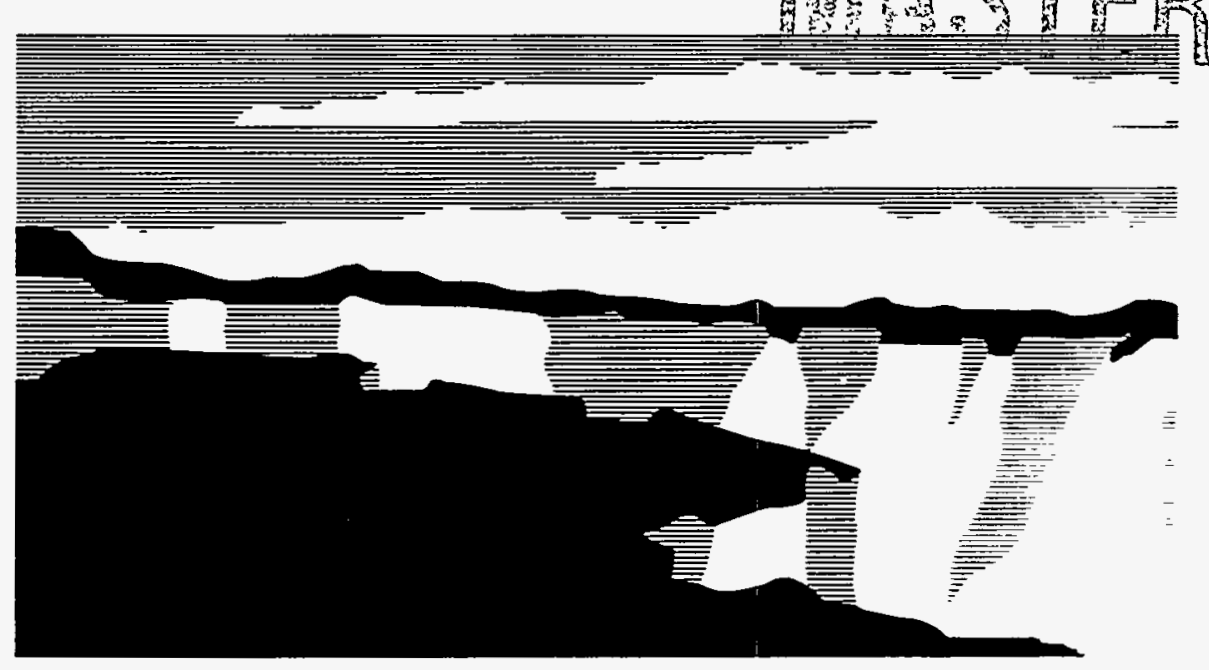

Los Alamos National Laboratory, an affirmative action/equal opportunity empldyer, is operated by the University of California for the U.S. Department of Energy under contract W-7405-ENG-36. By acceptance of this article, the publisher recognizes that the U.S. Government retains a nonexclusive, royalty-tree license to publish or reproduce the published form of this contribution, or to allow others to do so, for U.S. Government purposes. The Los Alamos National Laboraton requests that the publisher identify this anticle as work performed under the auspices of the U.S. Department of Energy. 


\section{DISCLAIMER}

Portions of this document may be illegible in electronic image products. Images are produced from the best available original document. 


\title{
CALIBRATION AND USE OF A RUGGED NEW PIEZORESISTIVE PRESSURE TRANSDUCER*
}

\author{
R. A. Lucht and J. A. Charest \\ Los Alamos National Laboratory, MS C920, Los Alamos, NM 87545 \\ Dynasen, Inc., 20 Arnold Place, Goleta, CA 93017
}

\begin{abstract}
A new 50-ohm piezoresistive pressure gauge has been developed and calibrated in the range 0 to 4.0 GPa. This "pinducer" consists of one half of a $100 \mathrm{ohm}$, one quarter watt, carbon composition resistor mounted coaxially at the end of a small brass tube. Three techniques have been used to calibrate this new gauge. Good agreement is found between all calibration data, and a smooth curve is fit through all resistance change versus pressure data up to $1.5 \mathrm{GPa}$. The gauges exhibit rise times of about $0.5 \mu \mathrm{s}$. They offer advantages in nggedness, cost, and flexibility of application. The pinducer can be successfully used in divergent flows, harsh environments, and positions where lead protection would be impossible with thin-film gauges. A unique application is demonstrated.
\end{abstract}

\section{INTRODUCTION}

With the end of the cold war, emphasis shifted from the design of new weapons and munitions to the safety of the existing stockpile. This change has renewed interest in existing long-term programs and produced several new programs to study delayed transition-to-detonation (XDT) and deflagration-todetonation transition (DDT). XDT has been observed in propellants in shotgun and card gap tests $(1,2)$, and DDT in propellants (3), and granulated explosives (4). In many cases, XDT involves an insult with damage to the reactive material, resultant heating, ignition of burning, and DDT.

Whether pure DDT or XDT, the initiation mechanisms often involve relatively slow buildup of pressure from buming followed by the coalition of stress waves into a shock wave that can eventually run to detonation. Theoretical understanding of the details of these processes and accurate modeling of accident scenarios leading to XDT or DDT, requires measurement of the temporal change of pressure over a variety of time scales and at numerous positions. The measurements are complicated by the fact that the pressures often start quasi-statically and transition to waves with highly multi-dimensional flow. Thus, thin-film gauges such as carbon or manganin cannot be used because of the complicated flow and because they and their leads are not robust

\footnotetext{
*This work supported by the US Department of Energy.
}

enough to survive the duration and violence of the experiments. While gauges such as piezoelectric quartz gauges could be used in these applications, they often have prohibitively large (and thus slow) active regions and they are quite expensive for onetime use. These needs have driven the development of the "pinducer".

\section{DESIGN}

The term pinducer implies the combination of devices known as pressure transducers and time of arrival pins such as capped or ionization pins. Pinducers work like pressure transducers, but are shaped like and have the convenience features of pins. The pressure sensing element is one half of a $100 \Omega, 1 / 4$-watt resistor, manufactured by Mouser Corp. The details of its construction are shown in Fig. 1. The lead wire from the carbon composition resistor half is connected to the center conductor of the coaxial pin arrangement. Copper is vapor plated

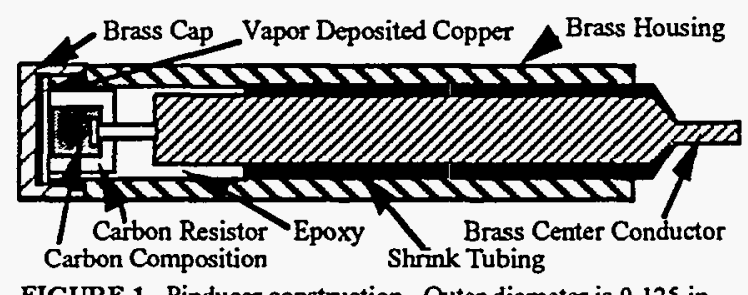

FIGURE 1. Pinducer construction. Outer diameter is 0.125 in. 
out on the cut surface of the resistor and provides a connection to the outer conductor, a brass tube housing, of the coax. This design builds upon the use of carbon composition resistors by Ginsberg and Asay (5) to measure pressure in multi-dimensional flows. Like other carbon gauges, its resis-tance decreases as the pressure increases. Because the active element is basically a small, right-circular cylinder of length about equal to diameter, it is quite insensitive to flow direction and planarity in shock pressure detection. However, its size does limit the time resolution to that required for it to reverberate to pressure equilibrium with its surroundings.

\section{CALIBRATIONS}

Typically, the development of a new gauge such as carbon film or manganin (6) has taken many years. Pinducers have only been available for about one year and some improvements could probably be made; however, a reasonable calibration curve has already been achieved using three very different types of apparatus.

The first apparatus generated a quasi-static pressure in the 0 to $0.2 \mathrm{GPa}$ range and is shown schematically in Fig. 2. It consisted of a heavy wall steel cylinder, a steel piston with two circumferential o-rings, and two gauge ports. One port fit a PCB Piezotronics Co. transducer with a 0.07315 $\mathrm{GPa} /$ volt calibration. The other port had 1/4-in. pipe threads to fit a 1/8-in. Swagelok fitting. The pinducer was held in this with nylon ferrules.

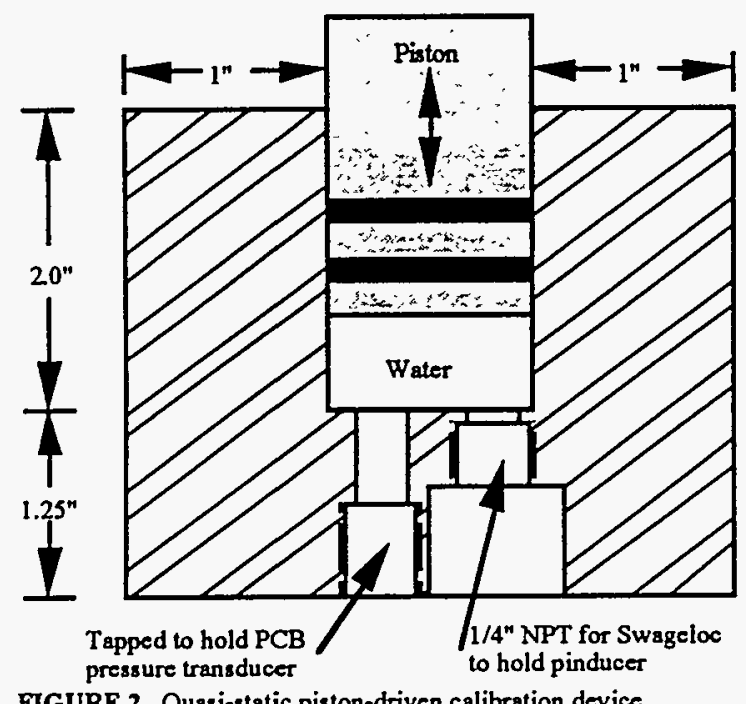

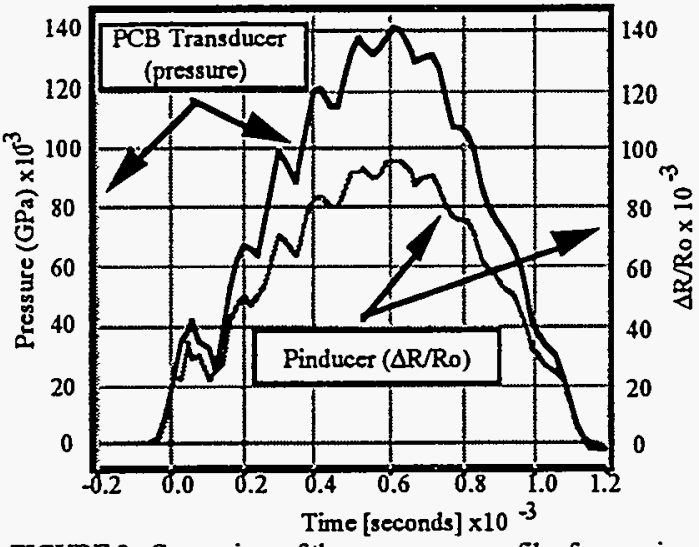

FIGURE 3. Comparison of the gauge wave profiles from a pinducer and a PCB Inc. transducer in the piston-driven quasi-static calibration test.

The piston was struck with a heavy hammer and the gauge's outputs were recorded on a LeCroy 9400 digital oscilloscope. Typically a standard pulsedWheatstone bridge-power supply would be used with a thin film, $50-\Omega$ gauge in a shock experiment. Because the piston-driven system produced gauge wave profiles of $1 \mathrm{~ms}$ or longer, a $9 \mathrm{v}$ direct-current power supply with a $50 \Omega$ viewing resistor was used with $\mathrm{I} M \Omega$ termination at the oscilloscope. An example of the results is shown in Fig. 3. After each test the resistance of the pinducer was measured, and if it had not changed, an additional test was run. Each pinducer was typically capable of withstanding many such tests before damage and permanent resistance change occurred, an example of the pinducer's robust construction. The data produced in this calibration consist of 58 points from 4 different pinducers. A quadratic least squares fit (LSF) with a zero intercept yields:

$$
\mathrm{P}(\mathrm{GPa})=1.318\left(\Delta \mathrm{R} / \mathrm{R}_{0}\right)+2.202\left(\Delta \mathrm{R} / \mathrm{R}_{0}\right)^{2}
$$

The scatter in the data is remarkably small and show that at least in this regime, the pinducer response is quite reproducible.

Four calibration points were generated with the gas gun at Dynasen in the 0.2 to $0.8 \mathrm{GPa}$ range. The calibration system used a 1/2-in.-thick PMMA flyer impacting a 1-in.-thick PMMA target with two embedded pinducers. The top of the pinducers were flush with the PMMA impact interface, and two carbon-film gauges were placed on this interface to produce reference peak pressures and wave forms. In addition, several different makes and sizes of 


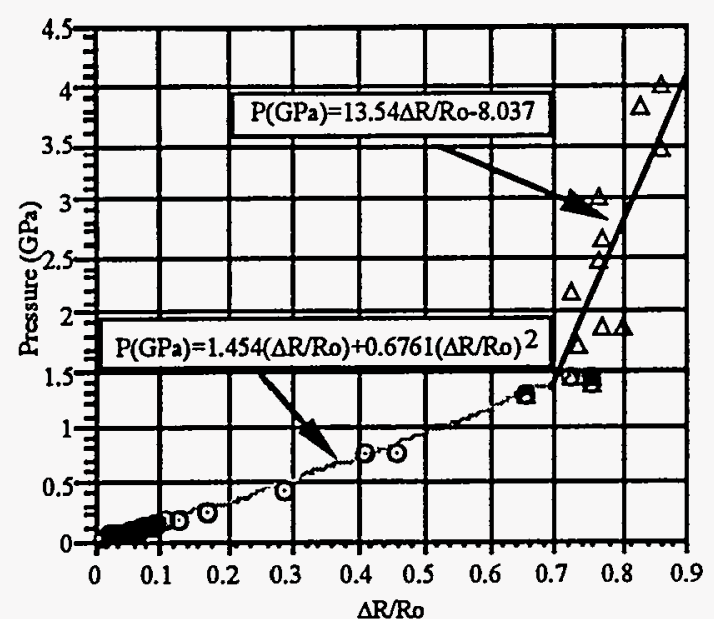

FIGURE 4. Pressure versus time plot of all calibration dat2. The quadratic LSF is to all data below $1.5 \mathrm{GP}_{2}$, and the linear LSF is to all data above $1 \mathrm{GPa}$.

resistors were tried in the pinducers. It was concluded that 1/8-watt resistors did not reproduce the waveform adequately, and 1/4-watt Mouser brand resistors produced slightly better results than Allen Bradley.

The third set of calibration data were generated using explosive plane-wave generated shock waves. Five experiments produced 16 calibration points in the range 0.7 to $4.0 \mathrm{GPa}$. Four of the shots used Detasheet $^{\mathrm{TM}}$-nitroguanidine (NQ) plane-wave lenses to drive shocks into PMMA-copper, aluminumpolymethyl methacrylate (PMMA), aluminumcopper, and PMMA-PMMA stacks of material. Reference gauges were carbon film in all cases. Some measurements were made at the interfaces of different materials, while others were made between layers of identical material. The fifth shot used a calcitol- PBX 9501 plane-wave lens, NQ driver, and an aluminum-PMMA-PBX9501 material stack. Measurements were made between the PMMA and PBX 9501. All the data from the three sources are shown in Fig. 4.

At the higher shock pressures there is evidence of ring up in the pinducers to come to the equilibrium pressure with its surroundings. An example of this can be seen in Fig. 5 where the response of a pinducer is compared to that of a carbon-film gauge. This is a result from the experiment where a NQ plane wave was used to drive a low-level shock into PMMA. It is also evident from Fig. 5 that the rise time of the pinducer $(-0.5 \mu \mathrm{s})$ is slower than that of the carbon film. The response to the trailing rarefaction is also somewhat different, with the carbon film response falling off faster than that of the

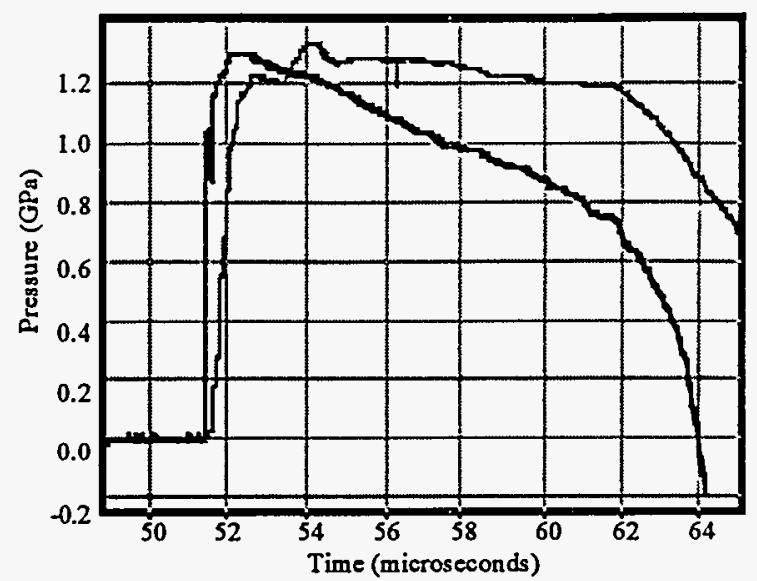

FIGURE 5. Comparison of the shock response of a carbon-film gauge and a pinducer. The experiment used a NQ plane-wave lens to drive a low-pressure shock into PMMA.

pinducer. Because calibration of pressure drops ater shocks is so difficult, it is impossible to estimate which, if either, gauge response is correct in this regime.

It is clear from Fig. 4 that pinducers are reliable from $0 \mathrm{GPa}$ to about 1.0 to $1.5 \mathrm{GPa}$. At pressures above $1.5 \mathrm{GPa}$, there is very little change in resistance for significant changes in pressure. Thus, it is difficult to reliably measure the difference between a 2.0-GPa shock and a 4.0-GPa shock. Up to $1.5 \mathrm{GPa}$, there is very little scatter in the data. The LSF of all this data predicts a pressure only $3 \%$ different from that predicted by the LSF in Equation (1) at $\Delta \mathrm{R} / \mathrm{Ro}=0.12$, the upper extent of this data. Thus, the data from the three types of calibration are consistent up to $1.5 \mathrm{GPa}$.

\section{APPLICATION AND RESULTS}

The pressure regime of interest in XDT or DDT processes is usually below $1.0 \mathrm{GPa}$. In these experiments, pressures often rise quasi-statically before generating a shock that can build and run to detonation. Thus, pinducers are ideal for these types of applications.

A set of experiments has been designed and executed at LANL to study the XDT potential of PBX 9501. The experiments were similar to those done by Chidester et al. (7). A schematic of the setup is shown in Fig. 6. A one piece stainless-steel holder with a 1.125-in.-deep, 6-in-diameter hole milled in it is used to hold a 1-in.-thick, 5.8-in.diameter piece of PBX 9501. The base of the holder was 0.5 -in.-thick stainless and had 0.125 -in. holes 


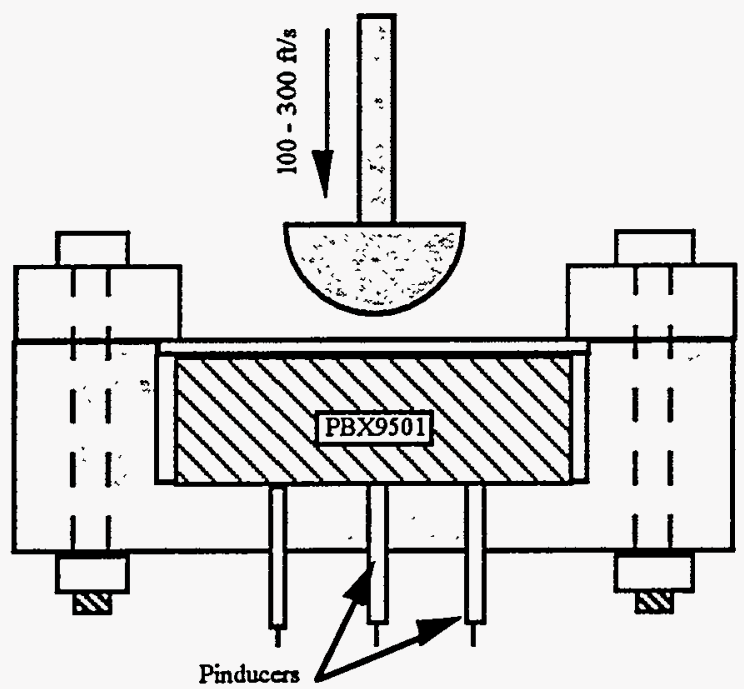

FIGURE 6. Schematic of spigot gun experiments. The PBX 9501 is held in a stainless-steel holder and is impacted by the mild steel projectile fired from the spigot gun.

drilled through it to accommodate the installation of pinducers. Above the PBX 9501 a 0.125-in-thick stainless-steel cover plate was held in position with a heavy retaining ring and eight bolts as shown. Several shots were fired without instrumentation in the targets to determine that the velocity threshold for violent reaction was in the 250-275-ft/s region.

In two recent experiments with instrumention, one with a projectile velocity of $235 \mathrm{ft} / \mathrm{s}$ showed no significant reaction, while the other at $265 \mathrm{ft} / \mathrm{s}$ reacted violently. Pressure versus time profiles are shown in Fig. 7 for the same pinducer position in

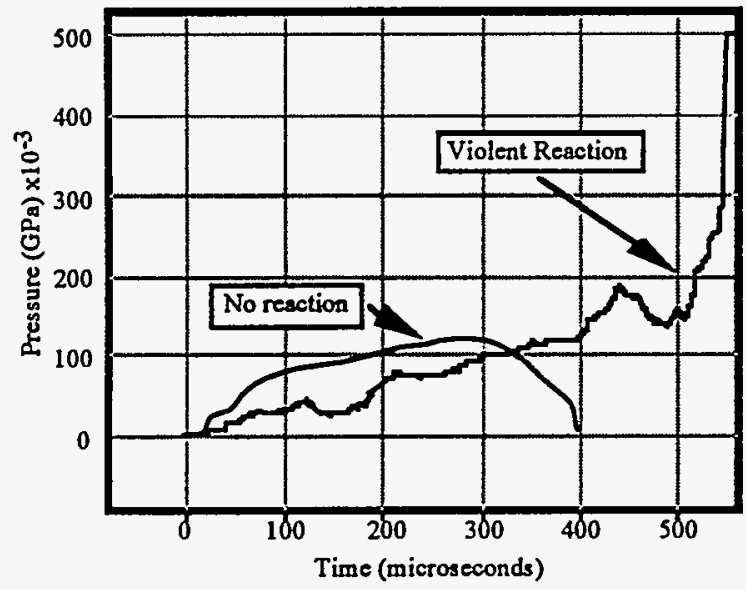

FIGURE 7. Wave profiles produced by pinducers from two experiments at slightly different impact velocities. the two different shots. In the lower velocity experiment the pressure rose slowly to about 0.1 $\mathrm{GPa}$ and then fell relatively slowly with a total duration of 400 microseconds. The pressure in the higher velocity experiment rose slowly for over 500 microseconds to about $0.2 \mathrm{GPa}$ and then rose rapidly to well beyond the peak pressures the pinducer is capable of measuring. The rapidity and extent of the rise indicate a strong shock or detonation wave. Magnetic analysis of the stainlesssteel target remains also showed some regions with strain typical of that produced by detonation waves. Comparison of the target remains from a promptly detonated system indicate that the PBX 9501 sustained at least a partial detonation in the violent reaction experiment.

\section{CONCLUSIONS}

The carbon composition resistor based pinducer has been shown to be capable of accurate pressure measurements in both the quasi-static and shock regimes up to pressures of $1.5 \mathrm{GPa}$. It is particularly useful for the hostile environments and long time regime experiments experienced in XDT and DDT processes.

\section{REFERENCES}

1. Jensen, R. C., Blommer, E. J., and Brown, B., "An Instrumented Shotgun Facility to Study Impact Initiated Explosive Reaction," in the Seventh Symposium on Detonation, 1981, pp. 299-307.

2. Keefe, R. L., "Delayed Detonation in Card Gap Tests," in the Seventh Symposium on Detonation, 1981, pp. 265-272.

3. Bernecker, R. R, Sandusky, H. W., and Clairmont, A. R. Jr., "Deflagration-to-Detonation Transition (DDT) Studies of a Double-Base Propellant," in the Eight Symposium on Detonation, 1985, pp. 658-668.

4. McAfee, J. M., Asay, B. W, Campbell, A. W., and Ramsay, J. B., "Deflagration to Detonation in Granular HMX," in the Ninth Symposium on Detonation, 1989, pp. 265-279.

5. Ginsberg, M. J., and Asay, B. W, "Commercial Carbon Composition Resistors as Dynamic Stress Gauges in Difficult Environments," Rev. Sci. Instrum. 62 (9), 2218-2227 (1991).

6. Rosenberg, T., Yaziv, D., and Partom, Y., "Calibration of Foil-Like Manganin Gauges in Planar Shock Wave Experiments," J. Appl. Phys. 51 (7), 3702-3705 (1980).

7. Chidester, S. K., Green, L. G., and Lee, C. G., "A Frictional Work Predictive Method for the Initiation of Solid High Explosives from Low-Pressure Impacts," Lawrence Livermore National Laboratory UCRL-JC-114186 (1993). 